\title{
SABERES DOS ENFERMEIROS SOBRE PREVENÇÃO DE INFECÇÃO DO SÍTIO CIRÚRGICO
}

\author{
Nurses' knowledge about prevention of surgical site infection \\ Conocimiento de enfermeras en la prevención de la infección quirúrgica del sitio
}

Karolayne Vieira de Souza ${ }^{1 *}$ (1), Solange Queiroga Serrano² (I)

RESUMO: Objetivo: Conhecer as experiências de enfermeiros sobre suas práticas na prevenção de infecção do sítio cirúrgico (ISC). Método: Estudo exploratório e qualitativo com enfermeiros da clínica cirúrgica geral de um hospital público do nordeste brasileiro. Entre dezembro de 2018 e janeiro de 2019 , realizou-se entrevista semiestruturada, gravada em áudio digital, com duração média de 20 minutos, para coleta de dados. Os depoimentos transcritos foram avaliados pela técnica de análise do discurso do sujeito coletivo. Resultados: Participaram nove enfermeiros, a maioria do sexo feminino, com idade média de 40,9 anos. Elencaram-se as seguintes categorias temáticas: medidas de prevenção contra ISC; assistência de enfermagem adequada na prevenção de ISC; equipe de enfermagem capacitada; adequadas condições de trabalho e de materiais; e treinamento contínuo. Conclusão: Observou-se preocupação em minimizar os riscos de ISC de pacientes por meio da adoção de ações preventivas, como lavagem das mãos, uso de equipamentos de proteção individual, troca de curativos diários com técnica asséptica, além do uso de insumos adequados, conhecimento técnico-científico harmonioso e estímulo do relacionamento eficaz entre a equipe. Palavras-chave: Infecção da ferida cirúrgica. Ferida cirúrgica. Contenção de riscos biológicos. Enfermagem. Cuidados perioperatórios.

ABSTRACT: Objective: To understand the experiences of nurses about their practices in preventing surgical site infection (SSI). Method: This is an exploratory qualitative study of nurses from the general surgery service of a public hospital in Northeastern Brazil. Between December 2018 and January 2019, a semi-structured interview was conducted for data collection. It was recorded in digital audio and lasted about 20 minutes. We analyzed the transcribed accounts using the Discourse of the Collective Subject technique. Results: Nine nurses participated in the study, mostly female, with a mean age of 40.9 years. The following thematic categories were listed: measures to prevent SSIs; adequate nursing care to prevent SSIs; qualified nursing staff; adequate working and material conditions; and ongoing training. Conclusion: We identified concern for minimizing risks of SSI in patients through the adoption of preventive actions, such as hand washing, use of personal protective equipment, daily dressing change using the aseptic technique, in addition to the use of adequate supplies, balanced technical-scientific knowledge, and effective team-building. Keywords: Surgical wound infection. Surgical wound. Containment of biohazards. Nursing. Perioperative care.

RESUMEN: Objetivo: Conocer las experiencias de las enfermeras sobre sus prácticas en la prevención de la infección del sitio quirúrgico (ISQ). Método: estudio exploratorio y cualitativo con enfermeras de la clínica quirúrgica general de un hospital público en el noreste de Brasil. Entre diciembre de 2018 y enero de 2019 , se grabó una entrevista semiestructurada, grabada en audio digital, con una duración promedio de 20 minutos, para la recopilación de datos. Los testimonios transcritos se evaluaron utilizando la técnica de análisis del discurso del sujeto colectivo. Resultados: participaron nueve enfermeras, la mayoría mujeres, con una edad promedio de 40,9 años. Se enumeraron las siguientes categorías temáticas: medidas preventivas contra SSI; asistencia de enfermería adecuada para prevenir SSI; equipo de enfermería capacitado; condiciones adecuadas de trabajo y materiales; y entrenamiento continuo. Conclusión: se observó preocupación por minimizar los riesgos de ISQ de los pacientes mediante la adopción de acciones preventivas, como el lavado de manos, el uso de equipos de protección personal, el intercambio de vendajes diarios con una técnica aséptica, además del uso de suministros adecuados, conocimiento armonioso técnico-científico y estimulando la relación efectiva entre el equipo. Palabras clave: Infección de la herida quirúrgica. Herida quirúrgica. Contención de riesgos biológicos. Enfermería. Atención perioperativa.

'Enfermeira pela Universidade de Pernambuco (UPE) - Recife (PE), Brasil.

Enfermeira; doutora em Saúde da Criança e do Adolescente pela Universidade Federal de Pernambuco (UFPE) - Recife (PE), Brasil.

*Autora correspondente: karolaynevieira316@gmail.com

Recebido: 20/06/2019 - Aprovado: 15/01/2020

DOI: $10.5327 / Z 1414-4425202000010003$ 


\section{INTRODUÇÃo}

As infecções relacionadas à assistência à saúde (IRAS) são consideradas um risco relevante à saúde dos usuários dos hospitais, contribuindo para o aumento da mortalidade, do tempo de internação e dos custos nessas instituições. No Brasil, na década de 1980, publicaram-se as primeiras portarias que priorizavam a prevenção e o controle das infecções hospitalares ${ }^{1}$.

Entre as IRAS, destacam-se as infecções do sítio cirúrgico (ISC) como uma das principais complicações relacionadas ao procedimento cirúrgico, com estimativa de 14 a 16\% entre as infecções nos serviços de saúde ${ }^{1}$. Entre os fatores de risco, estão tempo de internação pré-operatório acima de 24 horas, tempo de duração da cirurgia, potencial de contaminação da ferida operatória e classificação das condições clínicas dos pacientes (índice da American Society of Anesthesiologists - ASA $)^{2}$, obesidade, tabagismo, diabetes mellitus, uso de esteroides, imunossupressores, entre outros ${ }^{1}$.

Quando instalada a infecção, pode propiciar grandes encargos socioeconômicos com elevação da morbimortalidade e dos custos assistenciais, em virtude do aumento do tempo de internação, de antibioticoterapia associada, de cirurgias subsequentes para reconstituição do tecido, além do prolongamento do período de afastamento das atividades laborais e familiares do paciente ${ }^{2,3}$.

A equipe assistencial que acompanha o paciente no perioperatório tem a responsabilidade de, por meio do cuidado, minimizar as complicações relacionadas ao ato cirúrgico. Grande parte desses eventos adversos pode ser evitada, desde que as ações recomendadas e validadas sejam cumpridas, garantindo, assim, a segurança do paciente em todo o período intra-hospitalar ${ }^{3}$. Entre as recomendações adotadas para a prevenção e o controle das ISC, estão as medidas de precaução padrão (PP), utilizadas no contato com todos os pacientes, independentemente dos fatores de risco ou da doença de base, sempre que houver risco de contato com sangue e fluidos corporais ${ }^{4,5}$.

Entre as PP, destacam-se a higienização correta das mãos antes e depois do procedimento; o uso de luvas para redução de microrganismos, bem como a troca delas durante a realização de procedimentos entre um paciente e outro; o uso de aventais e máscaras ou proteção facial, para evitar o contato do profissional com fluidos do paciente, como sangue, líquidos corporais, secreção e excretas (exceto suor), pele não intacta e mucosa; e a prevenção de acidentes com material biológico 5 .

Para que as PP façam parte da rotina profissional da equipe de saúde, é necessário conhecimento técnico a respeito do assunto. Assim, esse tema deve ser frequentemente discutido e sua adesão incentivada. Vale salientar a importância da participação da equipe de enfermagem no processo de prevenção das ISC, já que ela presta assistência direta ao paciente ${ }^{6}$.

O enfermeiro deve avaliar os fatores predisponentes e de riscos à infecção e adotar medidas preventivas e educacionais para todos os sujeitos envolvidos, por meio de um processo de sensibilização coletiva, o que pode contribuir para a diminuição da ocorrência dessa complicação pós-cirúrgica ${ }^{3}$.

Ao considerar que o enfermeiro planeja, gerencia e avalia o cuidado, torna-se necessário avaliar as funções e as medidas implementadas por esse profissional, para o adequado controle das ISC, bem como para a promoção da segurança do paciente.

\section{OBJETIVO}

Conhecer as experiências de enfermeiros sobre suas práticas para a prevenção de ISC.

\section{MÉTODO}

Trata-se de uma pesquisa descritiva e exploratória, com abordagem qualitativa, desenvolvida na unidade de clínica cirúrgica geral de um hospital público de grande porte que realiza cerca de 800 cirurgias emergenciais e eletivas ao mês, no município do Recife, Pernambuco, Brasil.

Para isso, os indivíduos foram convidados a participar da investigação de acordo com os seguintes critérios de inclusão: ser enfermeiro e ter experiência na assistência ao paciente cirúrgico de no mínimo seis meses. Excluíram-se profissionais em licença de saúde, de maternidade ou em gozo de férias.

Os dados foram coletados em dezembro de 2018 e janeiro de 2019, por meio de entrevista individual semiestruturada, por meio das seguintes questões norteadoras:

- O que você entende por medidas de PP?;

- Quais são as ações prioritárias que você utiliza na prevenção da ISC?;

- Quais são as facilidades no desenvolvimento de suas ações?;

- Quais são as dificuldades no desenvolvimento de suas ações?;

- Quais medidas você toma para que a sua equipe estabeleça critérios de prevenção?

A entrevista foi feita em sala privativa, sem interrupções, com duração média de 20 minutos e gravada em áudio digital. 
O fechamento amostral deu-se por saturação, ou seja, quando não havia informação nova nos depoimentos.

Para garantir o anonimato, identificaram-se os participantes pela letra $E$, seguida da numeração arábica na ordem das entrevistas (E1, E2, E3...). Os dados transcritos foram avaliados pela técnica de análise do discurso do sujeito coletivo (DSC), que emprega figuras metodológicas (palavras-chave e ideias centrais), mediante o agrupamento dos depoimentos individuais com sentidos semelhantes, para serem categorizados, agregados em um discurso-síntese e redigidos na primeira pessoa do singular, representando a ideia do coletivo ${ }^{7}$. O programa de computação Qualiquantisoft ${ }^{\circledR}$ foi usado na tabulação dos dados ${ }^{8}$.

Todos os enfermeiros assinaram o Termo de Consentimento Livre e Esclarecido (TCLE), e o projeto de pesquisa foi submetido e aprovado pelo Comitê de Ética em Pesquisa sob o número 3.105.257, seguindo a Resolução n ${ }^{\circ} 466 / 2012$, do Conselho Nacional de Saúde.

\section{RESULTADOS}

Nove enfermeiros plantonistas (seis do horário diurno e três do noturno) compuseram a amostra, sendo sete do sexo feminino, com média de idade de 40,9 anos. O tempo de formação variou de um a 29 anos. Todos trabalhavam em mais de uma instituição, com carga média de trabalho de 42,22 horas semanais, e participavam de treinamentos.

Dos enfermeiros participantes da pesquisa, três possuíam apenas graduação em Enfermagem. Com relação a cursos lato sensu, encontraram-se especialistas em Saúde da Família (três enfermeiros), Emergência (dois), Administração dos Serviços de Saúde (dois), Enfermagem do Trabalho (um), Nefrologia (um), Educação em Saúde (um), Pediatria (um), Cirúrgica (um) e Obstetrícia (um). Vale ressaltar que alguns dos entrevistados tinham mais de uma especialização e apenas um especialização na área cirúrgica.

Da análise das entrevistas, por meio do DSC, surgiram seis categorias, relacionadas às boas práticas de controle das ISC. A seguir, apresentamos cada um dos discursos formulados, as respectivas discussões e a ideia central (IC).

\section{IC1/DSC1: Medidas de prevenção contra as infecções do sítio cirúrgico}

Ao responder sobre o conceito de medidas de PP, os enfermeiros definiram como principais ações que visam à prevenção de infecção a lavagem das mãos e o uso de equipamentos de proteção individual (EPI), gerando o seguinte discurso:
São as medidas que todo o profissional de saúde deve tomar para evitar a contaminação por bactérias que levam a infecções no contato com o paciente. Tenho que ter sempre como norma a lavagem das mãos antes e após o procedimento; o uso de EPIs como máscara, luva, óculos, capote e touca.

\section{IC2/DSC2: Assistência de enfermagem adequada na prevenção de infecções do sítio cirúrgico}

Quanto às ações prioritárias utilizadas na prevenção das ISC, os enfermeiros destacaram a lavagem das mãos, a troca diária de curativos com técnica asséptica e materiais corretos e a orientação do paciente para o autocuidado:

Como ações prioritárias na prevenção de infecções, eu lavo as mãos antes e após os procedimentos. Realizo a limpeza e troca de curativo da ferida operatória diariamente e sempre que necessário com os materiais adequados e técnica correta. Oriento o paciente e acompanhante quanto à sua higiene. Faço a manutenção de drenos e sondas.

Para responder à questão sobre as facilidades encontradas no desenvolvimento das ações preventivas, foram identificadas duas categorias temáticas, descritas a seguir.

\section{IC3/DSC3: Equipe de enfermagem capacitada}

Os entrevistados apontaram a atuação da enfermagem para a prevenção de ISC como uma atividade em equipe, bem como a relevância do seu conhecimento técnico na prestação da assistência, como explicitado no discurso:

Acredito que a maior facilidade é a questão do conhecimento. Você ter conhecimento do seu serviço e dos procedimentos. Ter uma equipe toda trabalhando em um só propósito e gostar de fazer o que faz.

\section{IC3/DSC4: Recursos materiais adequados para a assistência}

Para a qualidade da assistência, foram destacados também os materiais em quantidade suficiente como facilitadores de suas ações:

Uma facilidade é quando se tem o material correto e em quantidade adequada para a realização do procedimento. 
Às vezes a gente não vê a progressão daquela ferida cirúrgica pela escassez de produtos, como soluções, pomadas e curativos especiais. Isso, realmente, facilita o nosso trabalho e as medidas de precaução.

\section{IC4/DSC5: Adequadas condições de trabalho e de materiais}

Quanto às dificuldades na performance das suas ações, os entrevistados relataram que a falta de materiais impossibilita o cuidado adequado e que nessa situação o profissional tende a usar materiais inapropriados. A comunicação deficiente entre setores foi citada como outro fator prejudicial ao desenvolvimento da assistência:

A grande dificuldade é a falta de materiais, pois dificulta muito as nossas ações em relação à assistência ao paciente. Muitas vezes temos que fazer improviso, se virar nos 30, pois pode faltar água, sabão e papel toalha para a lavagem das mãos, por exemplo. A falta de comunicação entre profissionais dos diversos setores (farmácia, almoxarifado, [central de material e esterilização] $C M E$, [Comissão de Controle de Infecções Hospitalares] CCIH, entre outros) também prejudica as ações.

\section{IC5/DSC6: Treinamento contínuo em serviço}

Em relação às medidas estabelecidas para que a equipe previna as ISC, os participantes apontaram a importância do diálogo como ferramenta para a adesão da equipe a essas medidas. Esse diálogo é constituído de troca de informações e experiências. O treinamento e o uso de tecnologia digital foram citados como aliados na prevenção das ISC:

Geralmente eu utilizo o diálogo, a troca de informação $e$ de experiência entre funcionários para melhoria do conhecimento e, assim, colocar em prática nossos planos de ação de prevenção de infecções. Faço treinamento e sempre que temos dúvidas vou consultar na internet sobre o caso e repasso as informações para a equipe. Muita gente tem resistência ao uso de equipamentos de proteção, mas o que eu posso fazer é lembrar aos funcionários de se protegerem e ao seu paciente. Na rede privada você é punido pelo não uso de equipamento $e$ na rede pública não tem punição, não tem fiscalização.

\section{DISCUSSÃO}

Os procedimentos cirúrgicos são amplamente utilizados na realidade hospitalar. Apesar de todo o avanço em pesquisas e em tecnologia, os tratamentos cirúrgicos oferecem riscos ao paciente ${ }^{9}$. As ISC geram altos gastos financeiros, consequentes ao aumento do número das intervenções cirúrgicas e à complexidade desses pacientes. Estudos indicam que as ISC podem ser reduzidas desde que as ações estabelecidas nos protocolos e nas normas das instituições estejam baseadas em evidências ${ }^{10}$.

As medidas de PP são empregadas no controle das IRAS. Nessa casuística, as PP foram definidas como ações que visam evitar a contaminação por bactérias que levam a infecções por meio do contato com o paciente. O profissional deve estar paramentado, utilizando máscara, luvas, óculos, capote e touca. Outros estudos corroboraram com essa definição e também ressaltam que as medidas devem fazer parte do cotidiano dos profissionais na assistência aos pacientes ${ }^{6,11,12}$.

A lavagem das mãos antes e depois dos procedimentos foi a ação elencada como prioritária no controle de infecção. Ao considerar que as mãos dos profissionais são as principais responsáveis pela disseminação de microrganismos, higienizá-las é medida simples e eficaz para prevenir infecções associadas à assistência, com boa relação custo-benefício ${ }^{13,14}$. O profissional, ao aderir a essas medidas, contribui para o desenvolvimento da qualidade da assistência aos usuários, com benefícios na redução da morbimortalidade ${ }^{12}$. Outras ações citadas foram a troca de curativo e o manejo adequado de sondas e cateteres.

Com esses resultados, é possível perceber que as ações dos enfermeiros são gerais, sem levar em conta as especificidades de cada período operatório. Constatou-se conhecimento fragmentado por parte dos profissionais a respeito dos fatores que podem desenvolver complicações pós-cirúrgicas relacionadas ao pré e transoperatório, e que talvez estejam ligados à abordagem insatisfatória durante a graduação e atrelados à ausência de atualizações frequentes após a formação ${ }^{15}$.

Apenas um enfermeiro do setor possui especialização na área cirúrgica, o que pode nos remeter a uma cultura do não aproveitamento de especialistas em setores diferenciados, pois esse profissional seria mais adequado, em nível de conhecimentos específicos, quanto às relações características do trabalho sobre a assistência ao paciente cirúrgico. O especialista é capaz de dominar com rapidez e segurança as situações comuns ou inesperadas, com enfrentamento e resolutibilidade ${ }^{16}$.

Estudo realizado em um hospital-escola público de referência identificou os fatores de risco para ISC pertinentes 
ao pré-operatório, como tabagismo, etilismo, hipertensão e diabetes, os quais devem ser controlados antes da realização cirúrgica $^{15}$. No que diz respeito ao período transoperatório, os procedimentos classificados como cirurgias potencialmente contaminadas e cirurgias limpas foram apontados como os de maior prevalência de ISC, além do tempo cirúrgico prolongado e do uso de drenos. Por fim, no pós-operatório, elencaram-se a realização de cultura da secreção, os cuidados de enfermagem com drenos e curativos e o adequado controle das comorbidades para a redução das infecções ${ }^{15}$.

Entre os fatores facilitadores, foram mencionados conhecimento técnico agregado ao trabalho em equipe e materiais adequados para que se possa alcançar assistência efetiva e garantir a segurança do paciente. Ao considerar que o enfermeiro conduzirá intervenções realizadas pela equipe de enfermagem, é necessário que o profissional possua conhecimento teórico-prático e técnico-científico. Além desses fatores, é relevante que ele mostre iniciativa, interesse, persistência e liderança. Todas essas características são de suma importância para uma assistência de qualidade ${ }^{17}$.

Outros fatores facilitadores para assistência de qualidade, não citados pelos enfermeiros, são os protocolos de normas e rotinas adequados à realidade do serviço e a promoção da cultura de segurança do paciente por meio da educação permanente, pois ambos contribuem para a redução de eventos $\operatorname{adversos}^{18,19}$. Vale ressaltar ainda a participação ativa da CCIH no desenvolvimento dessas ações ${ }^{19}$.

Sobre as dificuldades, apontou-se a falta de materiais como prejudicial à assistência. Ambientes precários exercem influência negativa nas atividades realizadas pelos profissionais e provocam estresse relacionado à insatisfação no trabalho. Condições inadequadas de trabalho prejudicam a rotina do serviço, sobrecarregando o enfermeiro, ficando o cuidado direto ao paciente em segundo plano ${ }^{18}$. Estudo aponta que os profissionais de enfermagem estão expostos a diversos riscos ocupacionais, no que tange a estruturas, equipamentos, espaço físico e número limitado de trabalhadores ${ }^{20}$.

A comunicação deficiente também foi citada como fator dificultador para prestar o cuidado. Os profissionais no ambiente intra-hospitalar devem desenvolver comunicação efetiva entre os diversos setores para o bom desenvolvimento de ações de prevenção e controle das ISC. Outros estudos mostraram número insuficiente de profissionais, ausência de palestras e cursos, falta de padronização na assistência e vigilância epidemiológica como fatores que contribuem para o aumento de infecções ${ }^{18,19}$.

A atuação do enfermeiro na equipe de enfermagem foi apontada como fator pertinente. A equipe assistencial é indispensável no controle das infecções, uma vez que presta cuidados ao paciente durante todo o período de internamento hospitalar. Para que a prevenção seja alcançada, faz-se necessário que todos os envolvidos cumpram com as ações baseando-se em evidências ${ }^{3}$.

Os métodos educacionais citados, como treinamento, diálogo, troca de experiências e uso de inovações tecnológicas pela internet, auxiliam no cuidado de qualidade. Por meio da análise dos fatores de risco, o enfermeiro pode atuar ativamente na prevenção das ISC, utilizando métodos educacionais para que todos os indivíduos envolvidos pratiquem as medidas preventivas e, assim, diminuam essa grave complicação. O desafio está em os profissionais assumirem sua responsabilidade no processo de redução das infecções, já que ainda se mostram resistentes a aderir a práticas seguras e validadas ${ }^{3}$.

As limitações do estudo dizem respeito às respostas dadas pelos enfermeiros, que podem ter sido transmitidas de forma mais resumida, pois a entrevista ocorreu durante o horário de trabalho e os profissionais poderiam estar preocupados com a questão de que o tempo utilizado pudesse comprometer seu trabalho.

\section{CONSIDERAÇÕES FINAIS}

O desenvolvimento do presente estudo possibilitou conhecer as experiências dos enfermeiros na prevenção de ISC de um hospital público de referência do nordeste do Brasil. As ações elencadas pelos profissionais sobre sua prática para o controle das ISC foram lavagem das mãos, uso de EPI, troca de curativos, insumos adequados, conhecimento técnico-científico e bom relacionamento entre a equipe.

Visando à adequada prevenção das IRAS, especificamente das ISC, o enfermeiro tem papel importante, bem como a sua equipe, para garantir a segurança do paciente. A fim de que a assistência seja adequada, é preciso conhecimento técnico, baseando suas ações em evidências científicas.

Constatou-se que os enfermeiros sofrem diante das fragilidades relacionadas à estrutura do serviço e à carência de fundamentação teórica. Faz-se necessária a promoção de uma cultura de segurança do paciente. Assim, será possível distinguir as prováveis causas e permitir reflexões por parte da equipe de enfermagem, que, consequentemente, adotará medidas de prevenção de infecções e de diminuição de erros.

As autoras sugerem aprofundamento na temática, com base em evidências científicas, na prática assistencial do enfermeiro em unidades cirúrgicas. 


\section{REFERÊNCIAS}

1. Brasil. Ministério da Saúde. Agência Nacional de Vigilância Sanitária. Critérios diagnósticos de infecções relacionadas à assistência à saúde. Brasília: ANVISA; 2017.

2. Carvalho RLR, Campos CC, Franco LMC, Rocha AM, Ercole FF. Incidência e fatores de risco para infecção de sítio cirúrgico em cirurgias gerais. Rev Latino-Am Enferm. 2017;25:e2848. http:// dx.doi.org/10.1590/1518-8345.1502.2848

3. Santana CA, Oliveira CGE. Assistência de enfermagem na prevenção de infecções de sítio cirúrgico: uma revisão integrativa da literatura. Rev Eletrôn Atualiza Saúde [Internet]. 2015 [acessado em 22 mar. 2018];1(1). Disponível em: http://atualizarevista.com. br/wp-content/uploads/2015/01/ASSISTENCIA-DE-ENFERMAGEMNA-PREVENCAO-DE-INFECCOES-DE-SITIO-CIRURGICO-UMAREVIS\%C3\%830-INTEGRATIVA-DA-LITERATURA-REVISTAATUALIZA-SAUDE-N1-V1.pdf

4. Reis RG, Rodrigues MCS. Infecção de sítio cirúrgico pós-alta: ocorrência e caracterização de egressos de cirurgia geral. Cogitare Enferm [Internet]. 2017;22(4):e51678. [acessado em 22 jan. 2019]. Disponível em: http://docs.bvsalud.org/biblioref/2017/12/876610/51678220463-1-pb.pdf

5. Garcia L, César ICO, Braga CA, Souza GAAD, Mota ÉC. Perfil epidemiológico das infecções hospitalares por bactérias multidrogarresistentes em um hospital do norte de Minas Gerais. Rev Epidemiol Control Infect. 2013;3(2):45-9. http://dx.doi.org/10.17058/ reci.v3i2.3235

6. Lacerda MKS, Souza SCO, Soares DM, Silveira BRM, Lopes JR. Precauções padrão e precauções baseadas na transmissão de doenças: revisão de literatura. Rev Epidemiol Control Infect [Internet]. 2014 [acessado em 5 fev. 2019];4(4):254-9. Disponível em: https://online.unisc.br/seer/index.php/epidemiologia/article/ viewFile/4952/3985

7. Figueiredo MZA, Chiari BM, Goulart BNG. Discurso do Sujeito Coletivo: uma breve introdução à ferramenta de pesquisa qualiquantitativa. Distúrb Comum [Internet]. 2013 [acessado em 11 fev. 2019];25(1):12936. Disponível em: https://revistas.pucsp.br/index.php/dic/article/ view/14931

8. Lefévre F, Lefévre AMC. Discurso do sujeito coletivo. Qualiquanti soft. Passo a passo. São Paulo: IPDSC; 2010.

9. Silva FG, Oliveira Junior NJ, Oliveira DO, Nicoletti DR, Comin $\mathrm{E}$. Analysis of adverse events in an outpatient surgical center. Rev SOBECC. 2015;20(4):202-9. http://dx.doi.org/10.5327/ Z1414-4425201500040004

10. Berríos-Torres SI, Umscheid CA, Bratzler DW, Leas B, Stone EC, Kelz RR, et al. Centers for Disease Control and Prevention Guideline for the Prevention of Surgical Site Infection, 2017. JAMA Surg [Internet]. 2017 [acessado em 6 fev. 2019]; 152(8):784-91 Disponível em: https:// jamanetwork.com/journals/jamasurgery/fullarticle/2623725 http:// dx.doi.org/10.1001/jamasurg.2017.0904
11. Dutra GG, Costa MP, Ott Bosenbecker E, Lima LM, Siqueira HCH, Cecagno D. Controle da infecção hospitalar: função do enfermeiro. Rev Cuidado é Fundamental Online [Internet]. 2015 [acessado em 15 set. 2019];7(1):215968. Disponivel em: https://www.redalyc.org/articulo.oa?id=505750945033 http://dx.doi.org/10.9789/2175-5361.2015.v7i1.2159-2168

12. Organização Pan-Americana da Saúde. Centro Latino-Americano de Perinatologia, Saúde da Mulher e Reprodutiva. Prevenção de infecções relacionadas à assistência à saúde em neonatologia. Montevidéu: CLAP/ SMR-OPS/OMS; 2016. (CLAP/SMR. Publicação Científica, 1613-03).

13. Belela-Anacleto ASC, Peterlini MAS, Pedreira MLG. Hand hygiene as a caring practice: a reflection on professional responsibility. RevBras Enferm [Internet]. 2017 [acessado em 8fev. 2019];70(2):442-5. Disponivelem: http://www.scielo. br/scielo.php?script=sci_arttext\&pid=S0034-71672017000200442\&lng=en http://dx.doi.org/10.1590/0034-7167-2016-0189

14. Luangasanatip N, Hongsuwan M, Lubell Y, Limmathurotsakul D, Srisamang P, Day NPJ, et al. Cost-effectiveness of interventions to improve hand hygiene in healthcare workers in middle-income hospital settings: a model-based analysis. J Hosp Infect [Internet]. 2018 [acessado em 9 fev. 2019];100:165-75. Disponivel em: https:// www.ncbi.nlm.nih.gov/pmc/articles/PMC6204657/pdf/main.pdf https://doi.org/10.1016/j.jhin.2018.05.007

15. Aguiar APL, Prado PR, Opitz SP, Opitz SP, Vasconcelos SP, Faro ARMC. Fatores associados à infecção de sítio cirúrgico em um hospital na Amazônia ocidental brasileira. Rev SOBECC. 2012;17(3):60-70.

16. Oliveira NB, Silva FVC, Assad LG. Competências do enfermeiro especialista em nefrologia. Rev Enferm UERJ. 2015;23(3):375-80. http://dx.doi.org/10.12957/reuerj.2015.9789

17. Püschel VAA, Costa D, Reis PP, Oliveira LB, Carbogim FC. Nurses in the labor market: professional insertion, competencies and skills. Rev Bras Enferm [Internet]. 2017 [acessado em 5 fev. 2019];70(6):12206. Disponível em: http://www.revenf.bvs.br/scielo.php?script=sci arttext\&pid=S0034-71672017000601220\&lng=pt http://dx.doi. org/10.1590/0034-7167-2016-0061

18. Sell BT, Amante LN, Martins T, Sell CT, Pinho FM, Silva R. Adverse events in a hospital surgical unit: a descriptive study. Rev SOBECC. 2016;21(3):146-53. http://dx.doi.org/10.5327/Z1414-4425201600030005

19. Claudino HG, Fonseca LCT. Infecção de sítio cirúrgico: ações preventivas da comissão de controle de infecção hospitalar. Rev Enferm UFPE [Internet]. 2011 [acessado em 12 mar. 2019];5(5):1180-6. Disponível em: https://periodicos.ufpe.br/ revistas/revistaenfermagem/article/viewFile/6844/6092 http:// dx.doi.org/10.5205/reuol.1302-9310-2-LE.0505201113

20. Shoji S, Souza NVDO, Farias SNP, Vieira MLC, Progianti JM. Proposals for improving working conditions at anout patient clinic: the nursing stand point. Esc Anna Nery [Internet]. 2016 [acessado em 4 fev. 2019];20(2):303-9. Disponível em: http://www.scielo.br/scielo. php?script=sci_arttext\&pid=S1414-81452016000200303\&lng=en http://dx.doi.org/10.5935/1414-8145.20160041 